Review Article

\title{
Diagnostic Accuracy of Monofilament Tests for Detecting Diabetic Peripheral Neuropathy: A Systematic Review and Meta-Analysis
}

\author{
Fengyi Wang, ${ }^{1,2}$ Jiaqi Zhang, ${ }^{3}$ Jiadan Yu, ${ }^{2}$ Shaxin Liu, ${ }^{1,2}$ Rengang Zhang, ${ }^{1,2}$ Xichao Ma, ${ }^{1,2}$ \\ Yonghong Yang, ${ }^{1,2}$ and Pu Wang ${ }^{4}$ \\ ${ }^{1}$ Rehabilitation Medicine Center, West China Hospital of Sichuan University, Chengdu, China \\ ${ }^{2}$ West China School of Medicine, Sichuan University, Chengdu, China \\ ${ }^{3}$ Department of Rehabilitation Sciences, The Hong Kong Polytechnic University, Hung Hom, Hong Kong \\ ${ }^{4}$ Rui Jin Hospital, Shanghai Jiao Tong University School of Medicine, Shanghai, China
}

Correspondence should be addressed to Yonghong Yang; nicole@scu.edu.cn and Pu Wang; wangpu_03@126.com

Received 19 April 2017; Accepted 6 July 2017; Published 8 October 2017

Academic Editor: Konstantinos Papatheodorou

Copyright (C) 2017 Fengyi Wang et al. This is an open access article distributed under the Creative Commons Attribution License, which permits unrestricted use, distribution, and reproduction in any medium, provided the original work is properly cited.

Objective. To systematically evaluate the diagnostic accuracy of monofilament tests for detecting diabetic peripheral neuropathy. Methods. We searched EMBASE (OvidSP), MEDLINE (OvidSP), the Cochrane Library, and Web of Science to identify diagnostic accuracy trials of monofilament tests for detecting diabetic peripheral neuropathy. We used a hierarchical summary receiver operating characteristics (HSROC) model to conduct the meta-analysis of diagnostic accuracy of monofilament tests for detecting diabetic peripheral neuropathy. Results. A total of 19 comparative trials met the inclusion criteria and were part of the qualitative synthesis. Eight trials using nerve conduction studies as the reference standard were selected for the metaanalysis. The pooled sensitivity and specificity of monofilament tests for detecting diabetic peripheral neuropathy were 0.53 ( $95 \%$ confidence interval (CI) 0.32 to 0.74 ) and 0.88 (95\% CI 0.78 to 0.94 ), respectively. The pooled positive likelihood ratio and negative likelihood ratio were 4.56 (95\% CI 2.93 to 7.10 ) and 0.53 (95\% CI 0.35 to 0.81 ), respectively. Conclusions. Our review indicated that monofilament tests had limited sensitivity for screening diabetic peripheral neuropathy. The clinical use of the monofilament test in the evaluation of diabetic peripheral neuropathy cannot be encouraged based on currently available evidence.

\section{Introduction}

Diabetes mellitus (DM) is one of the most common metabolic diseases worldwide. The incidence, prevalence, and importance of DM as a chronic disease are increasing [1]. In 2010, approximately 21 million US adults aged 20 years or older had total confirmed diabetes (i.e., self-reported diabetes or diagnostic levels for both fasting glucose and calibrated $\mathrm{HbAlc}$ ) [2]. Diabetic peripheral neuropathy (DPN) is one of the most common complications of DM. This problem is frequently associated with a loss of sensation in the foot and an increased incidence of foot ulcers [3, 4], resulting in foot infection and even amputation in individuals with DM in the late stage [5].
Early detection of DPN contributes to preventing of foot ulcers and amputations. Several methods are used to detect DPN, including quantitative sensory testing, physical examination scoring systems (e.g., the neuropathy disability score), nerve conduction studies (NCS), and electrodiagnostic tests [6-8]. NCS is regarded as the standard for DPN diagnosis $[9,10]$; however, this examination is too time-consuming, highly demanding, and expensive to be implemented in many primary care settings. Thus, a portable, reliable, and valid tool for detecting DPN is urgently needed.

Monofilament tests have been widely used in clinical practice for DPN screening owing to their availability and convenience [11]. As a quantitative sensory test, a monofilament is used to test a single point of touch pressure. A 
$5.07 / 10 \mathrm{~g}$ monofilament is used to screen for the presence or absence of protective sensation [12, 13]. Several studies have explored whether a monofilament test is a useful screening tool for the early detection of DPN. Therefore, we conducted this systematic review with meta-analysis to quantitatively evaluate the currently available evidence regarding the diagnostic accuracy of monofilament tests for DPN detection.

\section{Methods}

2.1. Data Sources and Searches. We searched EMBASE (OvidSP, 1976 to April 2016), MEDLINE (OvidSP, 1946 to April 2016), the Cochrane Library (issue 4, 2016), and Web of Science (1995 to April 2016) to identify diagnostic accuracy studies of monofilament tests for detecting DPN. Our search strategy was focused on monofilament tests, diabetic peripheral neuropathy, and diagnostic accuracy. See Supplementary Material Appendices 1-4 available online at https://doi.org/10.1155/2017/8787261 for a complete list of search strategies.

2.2. Selection of Studies. One author screened all titles and abstracts generated by the electronic database searches for relevance and selected all potentially eligible studies for review of the full-text articles. Two reviewers independently assessed the full-text articles according to the inclusion criteria and exclusion criteria. When it was necessary, a third arbitrator resolved any disagreements that remained after discussion between the two reviewers. The inclusion criteria were as follows: (1) the study examined the diagnostic accuracy of a monofilament test for detecting DPN, (2) the article was published in English, and (3) the study provided sufficient data. The exclusion criteria were as follows: (1) the study was performed on patients without DM or (2) the study was performed on patients who had visible ulcers.

2.3. Data Extraction. Two authors independently extracted the following data from each included study: first author, year of publication, sample size, mean age of the participants, description of the monofilament, sites and number, threshold of the monofilament test, reference standard, sensitivity, and specificity. A third arbitrator resolved any remaining disagreements that the two review authors could not resolve through discussion. If more than one threshold was published in primary studies, we reported the diagnostic accuracy under all thresholds. The present systematic review of all diagnostic accuracy studies for the monofilament was conducted irrespective of reference standard utilized, whereas the quantitative synthesis was confined to trials using NCS as the reference standard. Additional information was extracted from studies that used NCS as the reference standard to demonstrate the variation across each study.

2.4. Assessment of Methodological Quality. We assessed the methodological quality of the studies using Quality Assessment of Diagnostic Accuracy Studies (QUADAS-2; http://www.bris.ac.uk/quadas), which has been recommended by Cochrane to assess the quality of primary diagnostic accuracy studies [14]. QUADAS-2 consists of four domains: patient selection, index test, reference standard, and patient flow/timing. Each domain is assessed for any risk of bias; the first three domains are also assessed for any concerns regarding applicability (see Supplementary Material Appendix 5). The risk of bias and applicability was analyzed using RevMan 5.3.

2.5. Statistical Analysis. Because of varying reference standards enrolled in studies, we selected studies that used NCS as the reference standard for meta-analyses of its validity in diagnosing DPN $[9,10]$. The data on true positives, true negatives, false positives, and false negatives were calculated based on the data reported by each original study. And the sensitivity, specificity, diagnostic odds ratio, positive likelihood ratio $(\mathrm{LR}+)$, negative likelihood ratio (LR-), and their 95\% confidence interval (CI) were presented in the forest plots performed by Meta-DiSc. The pooled sensitivity, specificity diagnostic odds ratio, positive likelihood ratio $(\mathrm{LR}+)$, and negative likelihood ratio (LR-) of included studies were calculated by using a hierarchical summary ROC model (HSROC) conducted by Stata 12.0.

\section{Results}

3.1. Results of the Search. A total of 522 records were identified through the electronic searches of MEDLINE (OvidSP) $(n=125)$, EMBASE (OvidSP) $(n=173)$, Cochrane $(n=33)$, and Web of Science $(n=191)$. We excluded 283 duplicate records. Four studies were identified by scanning the reference lists of the identified studies. A total of 187 irrelevant records were excluded by reading the titles and abstracts. In total, 56 potentially relevant studies were identified for fulltext analysis. Of these, 11 studies were not diagnostic accuracy studies, 4 studies were performed on patients without DM, 8 studies did not use a monofilament test as the index test, 7 studies were conference address or posters, 2 studies were without sufficient information and data, another study used the same data set as another published paper, and 4 studies were not published in English. In total, 19 comparative studies [15-33] met all eligibility criteria and were selected for qualitative analysis. These studies were conducted from 1997 to 2015. See Figure 1 for the details of the study search and selection process.

3.2. Data Extraction and Management. Sensitivity and specificity were retrieved or calculated from data available in the primary studies. Tables 1 and 2 list the characteristics of the included studies. The total sample size of all 19 studies was 3566 subjects, and the mean age of the participants ranged from 42 to 65 years old. Of the 19 studies, 6 studies did not report the type of monofilament used and 13 clearly reported that Semmes-Weinstein monofilaments (SWF) were used as the index test tool. The most commonly used test site was the great toe (plantar surface or dorsal surface). Nine of the 19 studies used NCS as the reference standard; vibration perception threshold (VPT), neuropathy disability score, and the Michigan Neuropathy Screening Instrument (MNSI) were also used. In the 19 studies, the sensitivity of the monofilament test ranged from 0.06 to 0.99 and the specificity ranged from 0.455 to 1.00 . Table 3 shows the variations of 


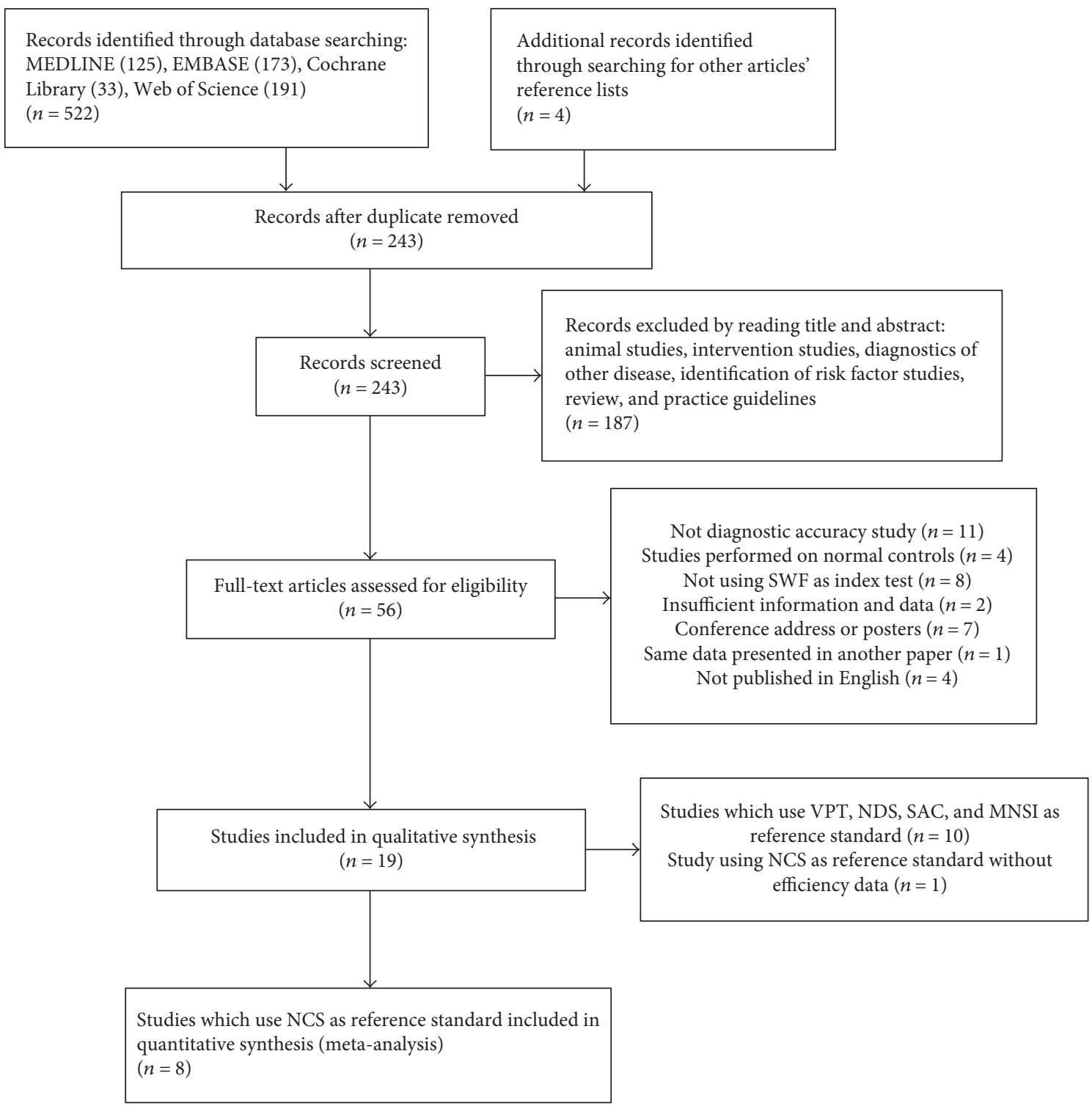

FIgURE 1: Flowchart of the study search and selection process.

the study set in the following: duration of DM, geographical distribution, and techniques of the monofilament test for studies using NCS as the reference standard.

3.3. Methodological Quality of the Included Studies. We assessed the methodological quality of the studies using QUADAS-2. The risk of bias and applicability concerns were analyzed using RevMan 5.3. Figures 2 and 3 show our assessment of each domain's risk of bias and applicability concerns for the included studies. In the selection of patients, three studies (Jayaprakash et al. [20], Paisley et al. [18], and Perkins et al. [29]) were labeled as low risk. The risk in the other 17 studies was unclear because they did not explicitly state whether a consecutive or random sample of patients was recruited or that the appropriate exclusions had been made.

Only five studies (Baraz et al. [32], Lee et al. [27], Perkins et al. [29], Pourhamidi et al. [31], and Rayman et al. [21]) had both a low risk of bias and low applicability concerns regarding the index test. It was unclear whether the index test results were interpreted without knowledge of the reference standard results in the other 15 studies. The reference standard domain in 10 studies (Bracewell et al. [23], Jayaprakash et al. [20], Kamei et al. [19], Lee et al. [27], McGill et al. [16], Nagai et al. [17], Najafi et al. [24], Paisley et al. [18], Shin et al. [34], and Valk et al. [15]) was unclear because the studies did not state whether the investigators performing the reference standard were blinded to the results of the index test (SWF). The risks for flow and timing domain in all studies were also unclear because either the interval between the index test and the reference standard was unknown or not all patients were included in the final analysis.

3.4. Meta-Analysis. Studies using NCS as the reference standard were included in the quantitative synthesis; however, one study (Ruhdorfer et al. [33]) was excluded because of 


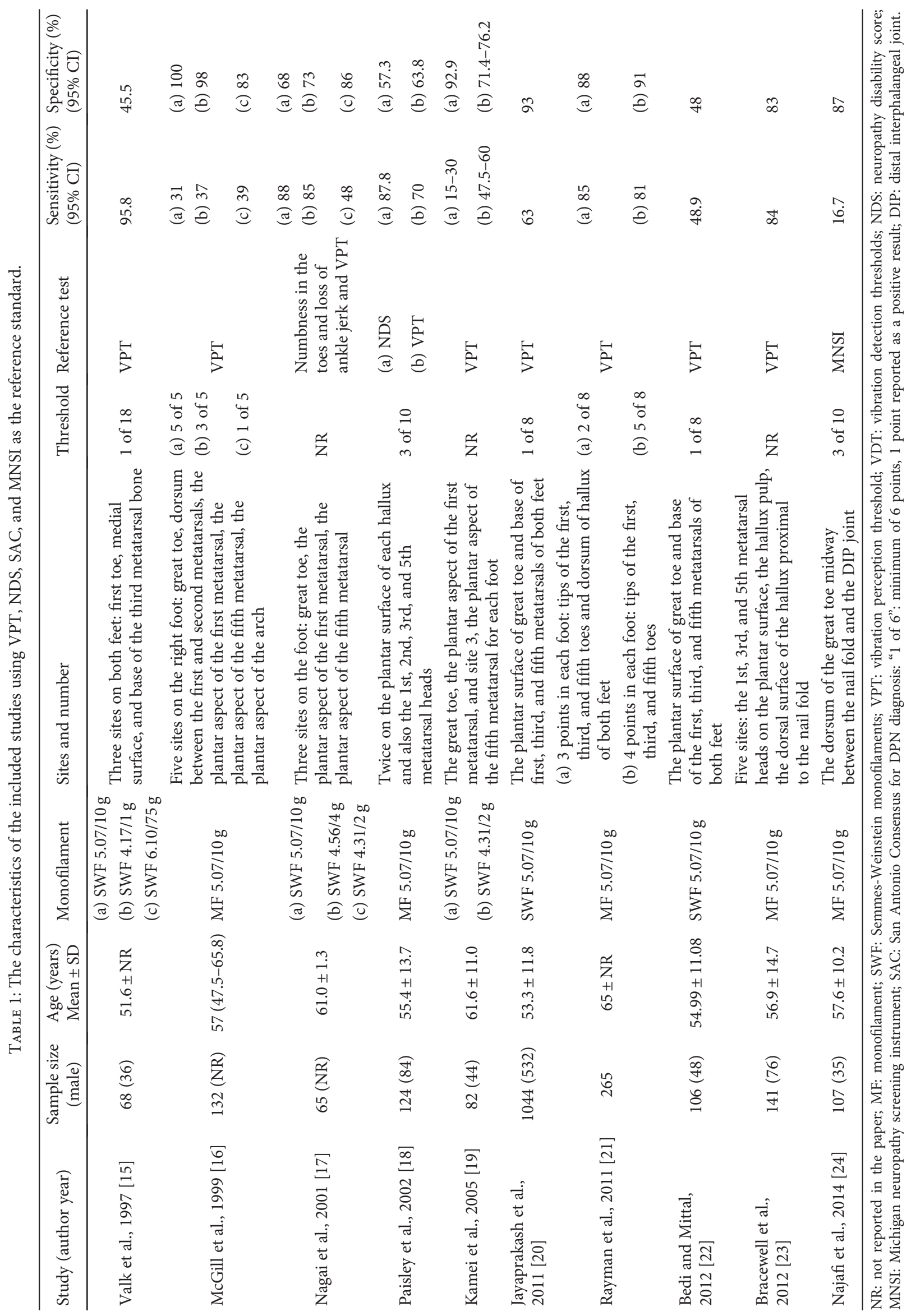




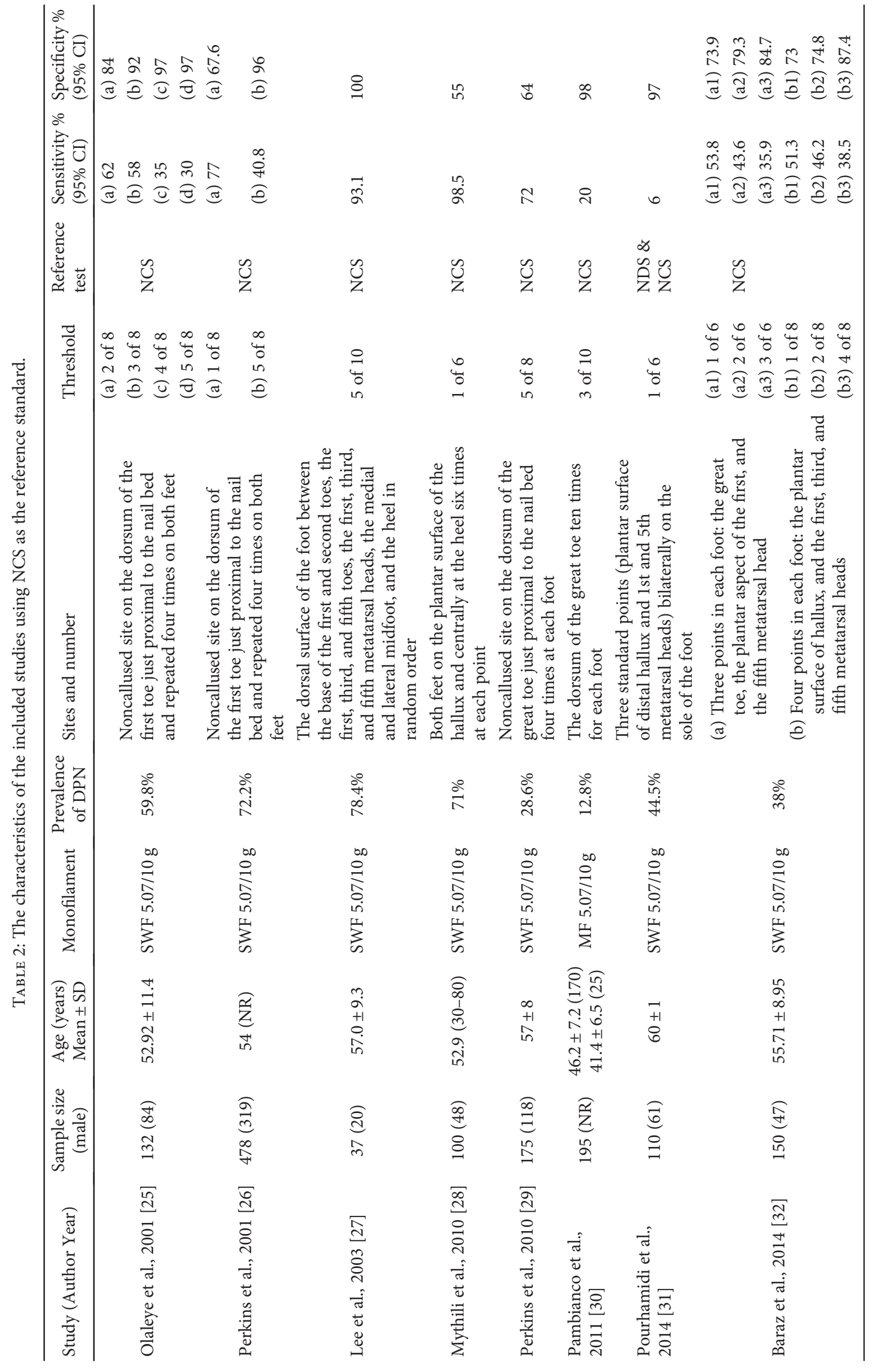




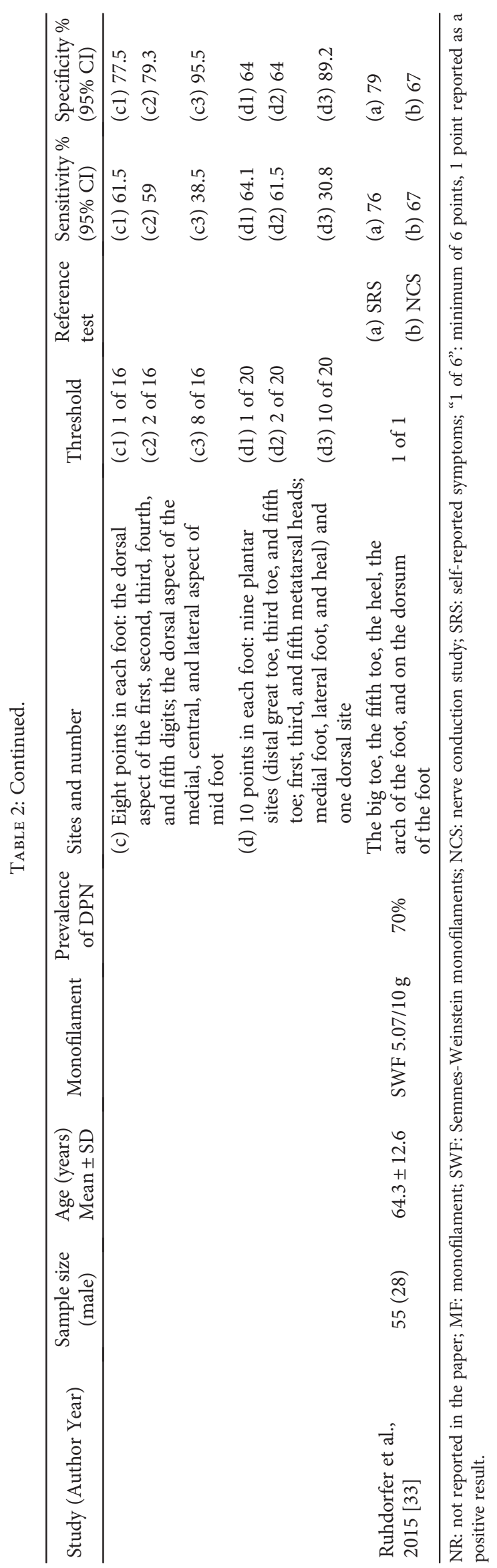


TABle 3: Moderator variables.

\begin{tabular}{|c|c|c|c|c|}
\hline Study (author year) & $\begin{array}{c}\text { Diabetes duration (years) } \\
\text { Mean } \pm \text { SD }\end{array}$ & $\begin{array}{c}\text { Type of the diabetes } \\
(\% n)\end{array}$ & Techniques & Geography \\
\hline \multirow[t]{2}{*}{ Olaleye et al., 2001 [25] } & $11.5 \pm \mathrm{NR}$ & Type $1(17.4 \%)$ & Yes-no & Canada \\
\hline & & Type $1(17.4 \%)$ & & \\
\hline \multirow[t]{2}{*}{ Perkins et al., 2001 [26] } & $12.53 \pm 11.47$ & Type $2(69.7 \%)$ & Yes-no & Canada \\
\hline & & NGT (12.9\%) & & \\
\hline Lee et al., 2003 [27] & $14.8 \pm 6.7$ & Type 2 & Yes-no & Korea \\
\hline Mythili et al., 2010 [28] & $6.9 \pm \mathrm{NR}$ & Type 2 & Yes-no & India \\
\hline Perkins et al., 2010 [29] & $13 \pm 9$ & Type $2(84 \%)$ & Forced choice $(0,0.5,1)$ & Canada \\
\hline \multirow{2}{*}{ Pambianco et al., 2011 [30] } & $33.6 \pm 5.2(n=25)$ & \multirow{2}{*}{ Type 1} & \multirow{2}{*}{ Yes-no } & \multirow{2}{*}{ USA } \\
\hline & $38.3 \pm 7.2(n=170)$ & & & \\
\hline \multirow{3}{*}{ Pourhamidi et al., 2014 [31] } & \multirow{3}{*}{$7.2 \pm 0.9$} & NGT (33\%) & \multirow{3}{*}{ Unknown } & \multirow{3}{*}{ Sweden } \\
\hline & & IGT (24\%) & & \\
\hline & & Type $2(43 \%)$ & & \\
\hline Baraz et al., 2014 [32] & $6.1 \pm 7.7$ & Type 2 & Yes-no \& point the site & Iran \\
\hline \multirow{2}{*}{ Ruhdorfer et al., 2015 [33] } & \multirow{2}{*}{$12.2 \pm 10.3$} & Type 1 (7.3\%) & \multirow{2}{*}{ Yes-no } & \multirow{2}{*}{ Austria } \\
\hline & & Type $2(92.7 \%)$ & & \\
\hline
\end{tabular}

NGT: normal glucose tolerance; IGT: impaired glucose tolerance; NR: not reported in the paper.

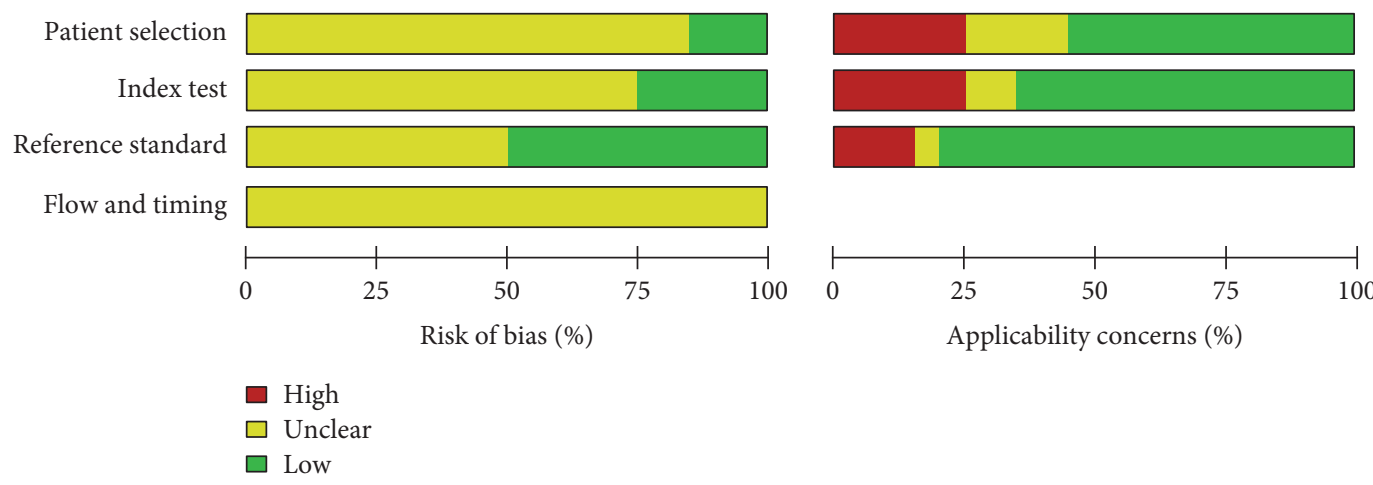

FIGURE 2: Risk of bias and applicability concerns: reviewers' judgments about each domain presented as percentages across included studies.

insufficient data. The remaining eight studies included in the statistical analysis had a total of 1377 participants, with a sample size ranging from 37 to 478 . Multiple thresholds were set in two studies (Olaleye et al. [25] and Baraz et al. [32]); we selected only the threshold that was closest to the threshold value ("half cutoff" threshold) used in other studies for the meta-analysis; therefore, a total of 12 groups of data were part of the meta-analysis.

The sROC analysis for the studies yielded an overall weighted area under the curve of 0.8158 (0.0364); the index $\mathrm{Q}^{*}$ value was $0.7498(0.035)$, which is a strong indicator $(0.7<\mathrm{ROC}=0.8158<0.9)$. A visual inspection of the forest plots shows large deviations, which indicate possible heterogeneity. Statistical tests such as chi-square, Cochran-Q, and the inconsistency index ( $I$-squared) can be used to quantify the amount of heterogeneity (see Figures 4-6). The forest plots show increasing sensitivities with decreasing specificities. Examination of the sROC curves for the studies reveals a "shoulder arm" plot, which indicates a threshold effect (Figure 7). Hence, a HSROC model was performed to pool the diagnostic parameters while considering the threshold effect. Under the HSROC model, the pooled sensitivity of studies was 0.53 (95\% CI 0.32 to 0.74$)$ and the pooled specificity was 0.88 (95\% CI 0.78 to 0.94$)$. The pooled diagnostic odds ratio (DOR) of 8.62 (95\% CI 4.69 to 15.84). The pooled positive likelihood ratio ( $\mathrm{LR}+$ ) and negative likelihood ratio (LR-) values were 4.56 (95\% CI 2.93 to 7.10$)$ and 0.53 (95\% CI 0.35 to 0.81 ), respectively (Table 4 , Figure 8 ).

Figures 4-6 showed the forest plot of the sensitivity, specificity, diagnostic odds ratio, LR+, and LR-, and the SROC and HSROC plot were presented in Figure 7 and Figure 8, respectively.

\section{Discussion}

Regular sensory examinations for people with DM are recommended by clinical guidelines [35]. Therefore, a portable and useful screen is necessary. Monofilament tests are one of the most common screening tools for DPN in primary care settings due to their availability. However, we found many 


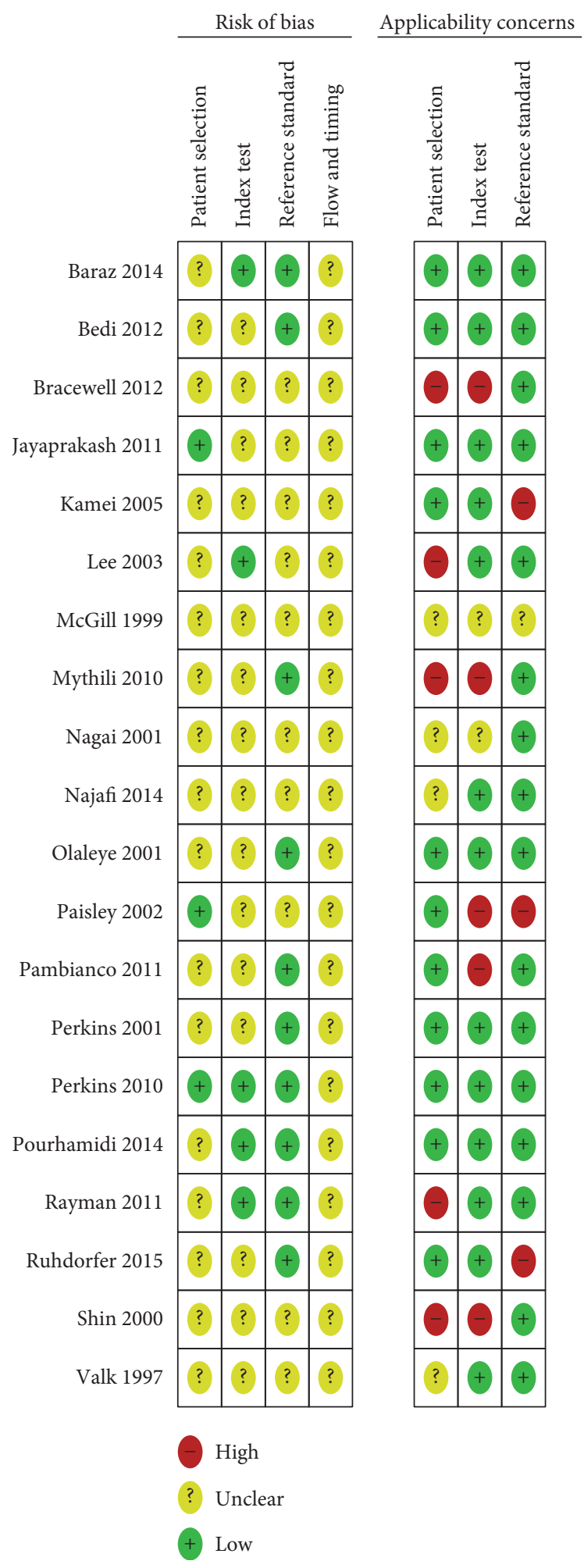

FIGURE 3: Risk of bias and applicability concerns: reviewers' judgments about each domain for each included study.

factors for consideration in the application of monofilament tests, and the role of monofilament tests in DPN diagnosis needs to be clarified.

A previous review [36] reported that the use of monofilament testing for the diagnosis of peripheral neuropathy has low sensitivity, which is in accordance with the main results of our study. Because of the variability of testing procedures, reference standards, and the application of the monofilament (number, site, and definition of thresholds), quantitative analysis was seldom used in previous reviews. We found a strong threshold effect among studies; therefore, the HSROC model was enrolled in the present study for pooled analyses, which jointly summarize pooled sensitivity and specificity while taking into account the threshold effect. When compared with NCS, our meta-analysis demonstrates that monofilament tests are fairly accurate for diagnosing DPN in individuals with DM. The pooled sensitivity and specificity values for studies using NCS as a reference standard were 0.53 and 0.88 , respectively; thus, the sole use of a monofilament test to screen for DPN cannot be recommended at this stage because of the test's low sensitivity. The pooled LR+ and LR- values were 4.56 and 0.53 , which are informative but not strong indicators for DPN confirmation or exclusion. A previous review [36] differed from the present review, in terms of their inclusion criteria, which was not limited to individuals with DM. For example, Shin et al. [34] enrolled patients who were referred to a foot clinic; this study was included in the previous review [36] but excluded by the present study because data that was specific to the cohorts of DM could not be extracted, and some studies [37-39] with healthy controls were excluded in our review as well. We also tried to exclude the studies of patients with visible ulcers, which can directly affect the diagnosis by monofilament tests or NCS; however, many studies did not report their inclusion criteria in detail to describe whether the participants have suffered visible foot ulcers or not.

Our findings are based on studies with low methodological quality (as identified by QUADAS-2); hence, many factors were labeled as being unclear. In practice, the monofilament test should directly follow or precede the reference standard test, with examiners blinded to the results. However, most of included studies did not report this information. An appropriate interval between the monofilament test and the reference standard is necessary because misclassification may occur due to recovery or deterioration of the targeted condition.

As part of a reliable clinical tool to assess changes in the protective sensation of the feet, the $5.07 / 10 \mathrm{~g}$ monofilament is the most commonly employed filament nowadays [40, 41]. However, Kamei et al. [19] suggested that SWF 4.31/2 g was a better diagnostic test for detecting DPN than 5.07/10 g monofilament, with sensitivity and specificity of 0.60 and 0.738 , respectively. The monofilament test sites also varied considerably across studies. An 18-site sensory examination [42] using $10 \mathrm{~g}$ monofilament that results in sensory change at three or more sites indicates actual change in the protective sensation of the feet. The most commonly used sites are on the great toe (plantar and dorsal), but there is no evidence to confirm that this is the most sensitive location for testing. Additionally, there is not a protocol for the threshold set of monofilaments to screen DPN, including sites and numbers. In some studies, a monofilament test was performed on different sites with different cutoff points 

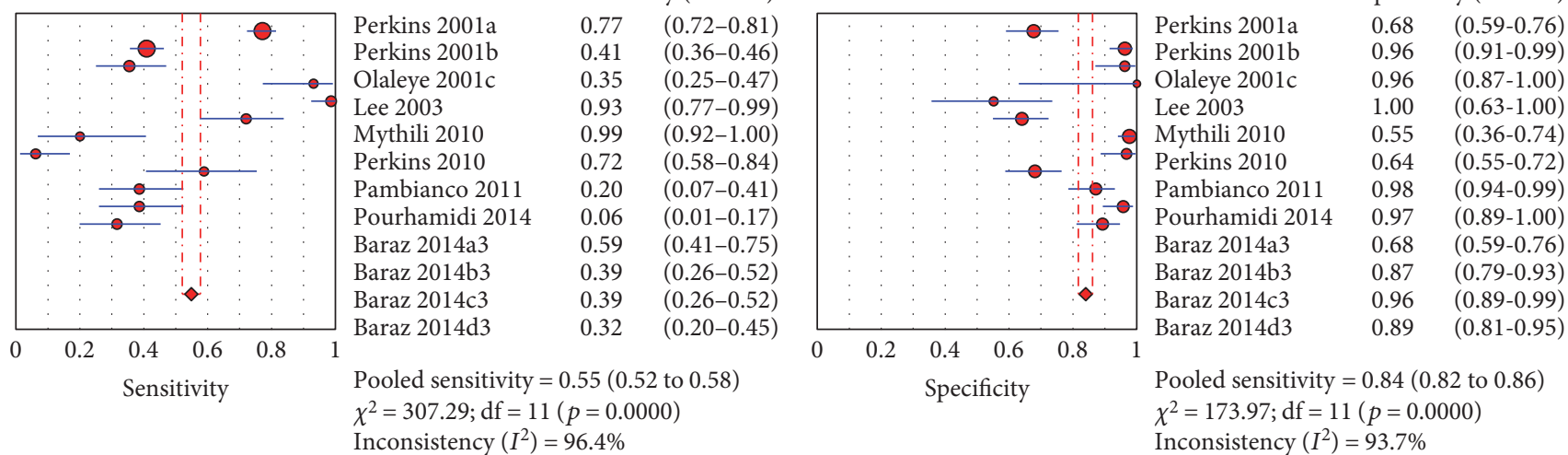

FIGURE 4: Forest plot of the sensitivity and specificity (red diamond) and its 95\% CI (blue horizontal line).

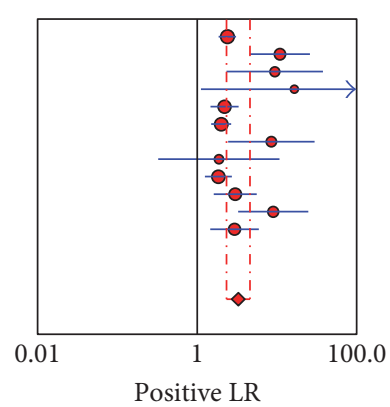

Random effects model

Pooled positive $\mathrm{LR}=3.27$ (2.34 to 4.58 )

Cochran $-\mathrm{Q}=39.89 ; \mathrm{df}=11(p=0.0000)$

Inconsistency $\left(I^{2}\right)=72.4 \%$

$\tau^{2}=0.1929$
Positive LR (95\% CI)

Perkins 2001a $\quad 2.38 \quad(1.85-3.07)$

Perkins 2001b $\quad 10.87 \quad(4.56-25.93)$

Olaleye 2001c $\quad 9.39 \quad(2.34-37.77)$

Mythili $2010 \quad 26.50 \quad(1.11-244.51)$

Perkins $2010 \quad 2.00 \quad(1.50-2.67)$

Pambianco $2011 \quad 8.50 \quad(2.45-29.55)$

Pourhamidi $2014 \quad 1.87 \quad(0.32-10.74)$

$(1.25-2.72)$

Baraz 2014b3 $\quad 2.99 \quad(1.61-5.57)$

Baraz 2014c3 $\quad 8.97 \quad(3.26-24.71)$

Baraz 2014d3 $2.94 \quad(1.46-5.91)$

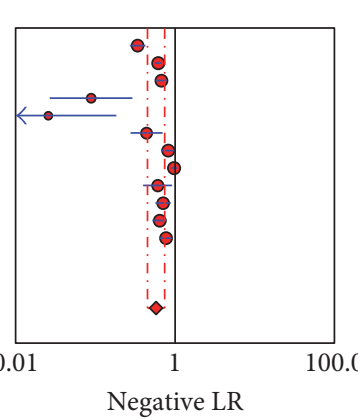

Random effects model

Pooled negative LR $=0.58$ ( 0.45 to 0.74 ) Cochran-Q $=196.19 ; \mathrm{df}=11(p=0.0000)$ Inconsistency $\left(I^{2}\right)=94.4 \%$ $\tau^{2}=0.1480$

FIGURE 5: Forest plot of the summary LR+ and LR-.

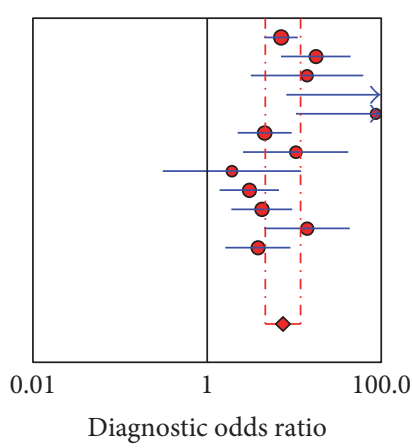

\begin{tabular}{lll} 
Perkins 2001a & 7.05 & $(4.53-10.96)$ \\
Perkins 2001b & 17.69 & $(7.06-44.35)$ \\
Olaleye 2001c & 14.00 & $(3.17-61.88)$ \\
Lee 2003 & 187.00 & $(8.16-4.287 .20)$ \\
Mythili 2010 & 86.15 & $(10.50-707.23)$ \\
Perkins 2010 & 4.57 & $(2.23-9.37)$ \\
Pambianco 2011 & 10.38 & $(2.57-41.83)$ \\
Pourhamidi 2014 & 1.92 & $(0.31-12.00)$ \\
Baraz 2014a3 & 3.05 & $(1.39-6.70)$ \\
Baraz 2014b3 & 4.24 & $(1.89-9.51)$ \\
Baraz 2014c3 & 13.99 & $(4.50-43.51)$ \\
Baraz 2014d3 & 3.83 & $(1.62-9.07)$ \\
\multicolumn{4}{l}{} \\
Random effects model \\
Pooled diagnostic odds ratio = 7.42 $(4.65$ to 11.83) \\
Cochran-Q $=27.71 ;$ df $=11(p=0.0036)$ \\
Inconsistency $\left(I^{2}\right)=60.3 \%$ \\
$\tau^{2}=0.3477$
\end{tabular}

FIgURE 6: Forest plot of the diagnostic odds ratio.

for positive results, with an increase of cutoff points to total points (e.g., 2/8 to 5/8); the sensitivity decreased, while the specificity increased, so the effects of bias should be considered. In addition, no clear difference in sensitivity was demonstrated by increasing the number of test points for
DPN screening. In Baraz et al.'s study [32], the sensitivity of the monofilament at three and four points is almost similar to its sensitivity at eight and ten points.

At present, monofilament tests have been already widely used and advocated for in many clinical guidelines. However, 


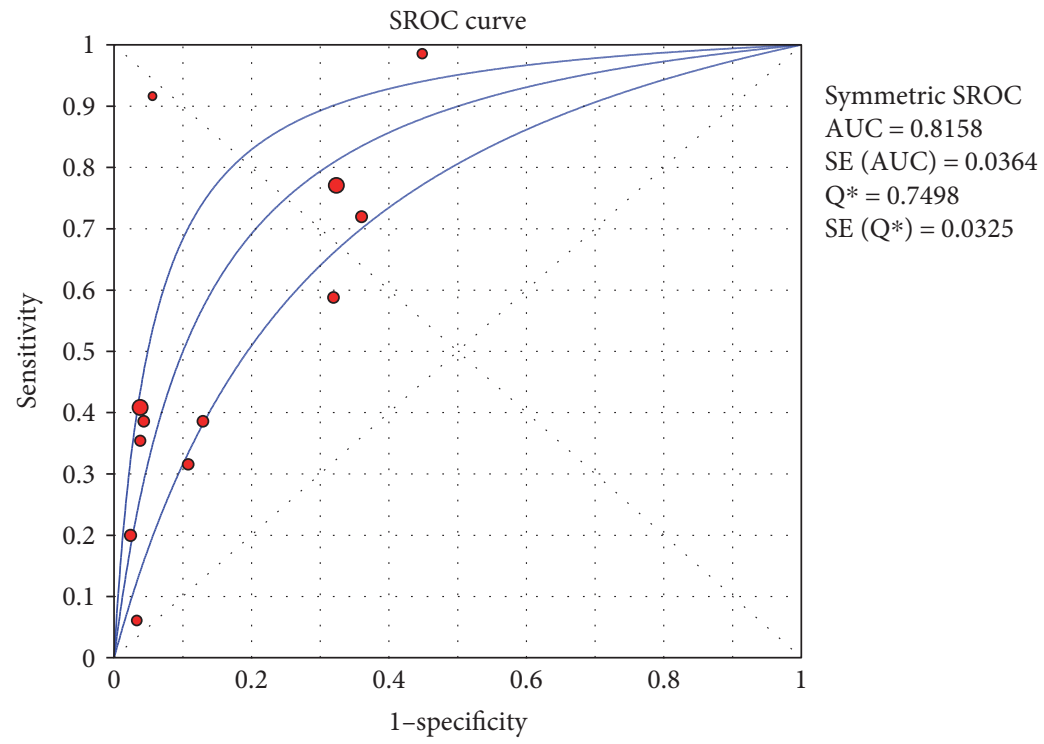

Figure 7: SROC with a 95\% confidence interval for monofilament tests in the diagnosis of DPN.

TABLE 4: Meta-analysis of diagnostic accuracy under the HSROC model.

\begin{tabular}{lcccc}
\hline & Pooled value & SE & \multicolumn{2}{c}{$95 \%$ CI } \\
\hline Sensitivity & 0.53 & 0.12 & 0.32 & 0.74 \\
Specificity & 0.88 & 0.04 & 0.78 & 0.94 \\
DOR & 8.62 & 2.68 & 4.69 & 15.84 \\
LR+ & 4.56 & 1.03 & 2.93 & 7.10 \\
LR- & 0.53 & 0.11 & 0.35 & 0.81 \\
\hline
\end{tabular}

SE: standard error; CI: confidence interval.

there is no consensus on the optimal location, number of sites, and threshold values for DPN diagnosis. Therefore, further research is needed to standardize the method for clinical practice. As indicated by our meta-analysis, heterogeneity exists among studies. Two main causes are indicated: (1) there were different clinical protocols for the application of monofilaments in DM and (2) the subjects may differ in age, severity of DM, or other confounding factors. SemmesWeinstein 5.07/10 g monofilaments (manufactured by North Coast Medical) were used in 13 of the 19 studies included in our review. The commercial manufacturing source was frequently identified in studies; however, the durability of monofilaments should be considered. Longevity and recovery testing suggest that each monofilament can be used with approximately 10 patients, with a period of 24 hours required between uses [43]. Additionally, changes in relative humidity and temperature may affect the physical properties of monofilaments [44]. These details were not provided in the included studies. In order to demonstrate the monofilament test's ability to guide clinical decision making and to improve patient outcome, the prognostic and predictive value of monofilament tests can and should be evaluated by diagnostic randomized controlled trial. However, this evidence is lacking as our research result.

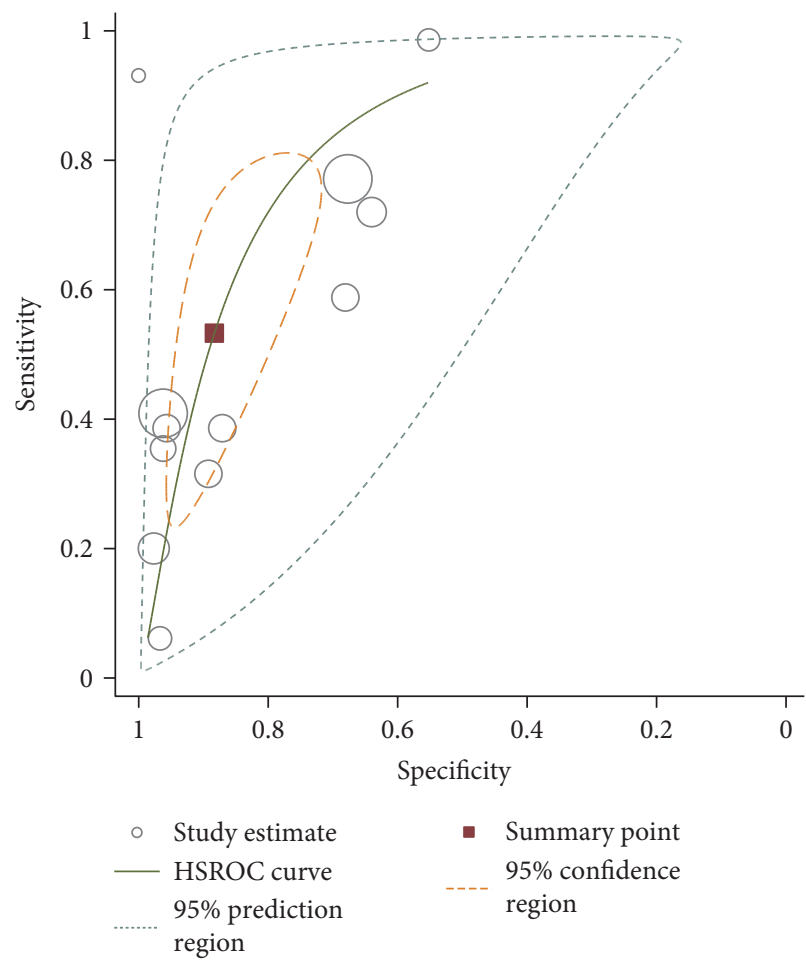

FIgURE 8: HSROC of the monofilament test for detecting DPN.

The present review has three main limitations. Firstly, our meta-analysis was performed based on a small number of studies with obvious heterogeneity. Although we used a HSROC model in our analysis, our conclusion should be interpreted with caution. Secondly, the protocol for using monofilament tests in DPN screening varied from study to study, which made it difficult to draw a firm conclusion at this stage. Thirdly, restricting the search to Englishlanguage publications may result in missing some relevant 
literature. Last but not least, we did not search grey literature sources and it can be acknowledged as a potential source of publication bias.

\section{Conclusion}

In summary, our study found that the 5.07/10 g SemmesWeinstein monofilament seemed to be a screen with limited sensitivity for DPN in primary care settings based on currently available evidence. Available studies with regard to the application of monofilament testing for DPN diagnosis varied greatly, and an optimal protocol for conducting monofilament tests in patients with DM is under exploration. Higher-quality studies on Semmes-Weinstein monofilament examination detecting DPN are needed.

\section{Conflicts of Interest}

No author of this study has a commercial/financial interest in or other relationship with manufacturers of pharmaceuticals, laboratory supplies, and/or medical devices or with commercial providers of medically related services.

\section{Acknowledgments}

The authors thank Fengjie Wang, who provided literature knowledge and useful information for this paper.

\section{References}

[1] G. Danaei, M. M. Finucane, Y. Lu et al., "National, regional, and global trends in fasting plasma glucose and diabetes prevalence since 1980: systematic analysis of health examination surveys and epidemiological studies with 370 country-years and 2.7 million participants," Lancet, vol. 378, no. 9785, pp. 31-40, 2011.

[2] E. Selvin, C. M. Parrinello, D. B. Sacks, and J. Coresh, "Trends in prevalence and control of diabetes in the United States, 1988-1994 and 1999-2010," Annals of Internal Medicine, vol. 160, no. 8, pp. 517-525, 2014.

[3] C. C. Lee, B. A. Perkins, S. Kayaniyil et al., "Peripheral neuropathy and nerve dysfunction in individuals at high risk for type 2 diabetes: the PROMISE cohort," Diabetes Care, vol. 38 , no. 5 , pp. 793-800, 2015.

[4] M. Janghorbani, H. Rezvanian, A. Kachooei et al., "Peripheral neuropathy in type 2 diabetes mellitus in Isfahan, Iran: prevalence and risk factors," Acta Neurologica Scandinavica, vol. 114, no. 6, pp. 384-391, 2006.

[5] Y. Yang, T. Ostbye, S. B. Tan, Z. H. Abdul Salam, B. C. Ong, and K. S. Yang, "Risk factors for lower extremity amputation among patients with diabetes in Singapore," Journal of Diabetes and its Complications, vol. 25, no. 6, pp. 382-386, 2011.

[6] R. Marchan, R. N. Ramkhalawan, K. Thannoo et al., "The diagnosis of diabetic peripheral neuropathy among type II diabetic patients: a challenge for primary healthcare physicians in Trinidad," The West Indian Medical Journal, vol. 61, no. 47, 2012.

[7] F. Forouzandeh, A. Aziz Ahari, F. Abolhasani, and B. Larijani, "Comparison of different screening tests for detecting diabetic foot neuropathy," Acta Neurologica Scandinavica, vol. 112, no. 6, pp. 409-413, 2005.
[8] S. K. Gupta, S. Popli, and S. Jha, "The role of quantitative sensory testing in asymptomatic diabetic neuropathy and its relationship with HBA1C," Value in Health Care, vol. 16, no. 3, pp. A170-A1A1, 2013.

[9] B. Perkins, "Diabetic neuropathy: update on methods for screening and diagnosis," Pediatric Diabetes, vol. 12, no. 2, 2011.

[10] J. Mason, C. O'Keeffe, A. McIntosh, A. Hutchinson, A. Booth, and R. J. Young, "A systematic review of foot ulcer in patients with type 2 diabetes mellitus. I: prevention," Diabetic Medicine, vol. 16, no. 10, pp. 801-812, 1999.

[11] R. A. Slater, S. Koren, Y. Ramot, A. Buchs, and M. J. Rapoport, "Interpreting the results of the Semmes-Weinstein monofilament test: accounting for false-positive answers in the international consensus on the diabetic foot protocol by a new model," Diabetes/Metabolism Research and Reviews, vol. 30, no. 1, pp. 77-80, 2014.

[12] N. K. Jamil, R. E. S. Anglin, D. L. Hunt, and A. Panju, "Does this patient with diabetes have large-fiber peripheral neuropathy?," Journal of the American Medical Association, vol. 303, no. 15 , pp. 1526-1532, 2010.

[13] L. S. Tan, "The clinical use of the $10 \mathrm{~g}$ monofilament and its limitations: a review," Diabetes Research and Clinical Practice, vol. 90, no. 1, pp. 1-7, 2010.

[14] P. F. Whiting, A. W. Rutjes, M. E. Westwood et al., "QUADAS-2: a revised tool for the quality assessment of diagnostic accuracy studies," Annals of Internal Medicine, vol. 155, no. 8, pp. 529-536, 2011.

[15] G. D. Valk, J. J. de Sonnaville, W. H. van Houtum et al., "The assessment of diabetic polyneuropathy in daily clinical practice: reproducibility and validity of Semmes Weinstein monofilaments examination and clinical neurological examination," Muscle \& Nerve, vol. 20, no. 1, pp. 116-118, 1997.

[16] M. McGill, L. Molyneaux, R. Spencer, L. F. Heng, and D. K. Yue, "Possible sources of discrepancies in the use of the Semmes-Weinstein monofilament. Impact on prevalence of insensate foot and workload requirements," Diabetes Care, vol. 22, no. 4, pp. 598-602, 1999.

[17] Y. Nagai, Y. Sugiyama, T. Abe, and G. Nomura, "4-g monofilament is clinically useful for detecting diabetic peripheral neuropathy," Diabetes Care, vol. 24, no. 1, pp. 183-184, 2001.

[18] A. Paisley, C. Abbott, C. van Schie, and A. Boulton, "A comparison of the Neuropen against standard quantitative sensory-threshold measures for assessing peripheral nerve function," Diabetic Medicine, vol. 19, no. 5, pp. 400-405, 2002.

[19] N. Kamei, K. Yamane, S. Nakanishi et al., "Effectiveness of Semmes-Weinstein monofilament examination for diabetic peripheral neuropathy screening," Journal of Diabetes \& its Complications, vol. 19, no. 1, pp. 47-53, 2005.

[20] P. Jayaprakash, A. Bhansali, S. Bhansali et al., "Validation of bedside methods in evaluation of diabetic peripheral neuropathy," Indian Journal of Medical Research, vol. 133, pp. 645649, 2011.

[21] G. Rayman, P. R. Vas, N. Baker et al., "The Ipswich touch test: a simple and novel method to identify inpatients with diabetes at risk of foot ulceration," Diabetes Care, vol. 34, no. 7, pp. 1517-1518, 2011.

[22] U. Bedi and G. Mittal, "Efficacy of simple bed side methods in diagnosing peripheral neuropathy in diabetic patients in comparison with vibration perception threshold," Research Journal of Pharmaceutical Biological and Chemical Sciences, vol. 3, no. 4, pp. 1178-1184, 2012. 
[23] N. J. Bracewell, F. L. Game, W. J. Jeffcoate, and B. E. Scammell, "Clinical evaluation of a new device in the assessment of peripheral sensory neuropathy in diabetes," Diabetic Medicine, vol. 29, no. 12, pp. 1553-1555, 2012.

[24] L. Najafi, M. E. Khamseh, M. Malek et al., "Rapid screening of diabetic polyneuropathy: selection of accurate symptoms and signs in an outpatient clinical setting," Acta Medica Iranica, vol. 52, no. 7, pp. 519-527, 2014.

[25] D. Olaleye, B. A. Perkins, and V. Bril, "Evaluation of three screening tests and a risk assessment model for diagnosing peripheral neuropathy in the diabetes clinic," Diabetes Research and Clinical Practice, vol. 54, no. 2, pp. 115-128, 2001.

[26] B. A. Perkins, D. Olaleye, B. Zinman, and V. Bril, "Simple screening tests for peripheral neuropathy in the diabetes clinic," Diabetes Care, vol. 24, no. 2, pp. 250-256, 2001.

[27] S. Lee, H. Kim, S. Choi, Y. Park, Y. Kim, and B. Cho, "Clinical usefulness of the two-site Semmes-Weinstein monofilament test for detecting diabetic peripheral neuropathy," Journal of Korean Medical Science, vol. 18, no. 1, pp. 103-107, 2003.

[28] A. Mythili, K. D. Kumar, K. A. Subrahmanyam, K. Venkateswarlu, and R. G. Butchi, "A comparative study of examination scores and quantitative sensory testing in diagnosis of diabetic polyneuropathy," International Journal of Diabetes in Developing Countries, vol. 30, no. 1, pp. 43-48, 2010.

[29] B. A. Perkins, A. Orszag, M. Ngo, E. Ng, P. New, and V. Bril, "Prediction of incident diabetic neuropathy using the monofilament examination: a 4-year prospective study," Diabetes Care, vol. 33, no. 7, pp. 1549-1554, 2010.

[30] G. Pambianco, T. Costacou, E. Strotmeyer, and T. J. Orchard, "The assessment of clinical distal symmetric polyneuropathy in type 1 diabetes: a comparison of methodologies from the Pittsburgh epidemiology of diabetes complications cohort," Diabetes Research and Clinical Practice, vol. 92, no. 2, pp. 280-287, 2011.

[31] K. Pourhamidi, L. B. Dahlin, E. Englund, and O. Rolandsson, "Evaluation of clinical tools and their diagnostic use in distal symmetric polyneuropathy," Primary Care Diabetes, vol. 8, no. 1, pp. 77-84, 2014.

[32] S. Baraz, K. Zarea, H. B. Shahbazian, and S. M. Latifi, "Comparison of the accuracy of monofilament testing at various points of feet in peripheral diabetic neuropathy screening," Journal of Diabetes and Metabolic Disorders, vol. 13, no. 1, p. 19, 2014.

[33] A. S. Ruhdorfer, M. Azaryan, J. Kraus et al., "Selecting a prospective test for early detection of diabetic polyneuropathy," Microsurgery, vol. 35, no. 7, pp. 512-517, 2015.

[34] J. B. Shin, Y. J. Seong, H. J. Lee, S. H. Kim, and J. R. Park, "Foot screening technique in a diabetic population," Journal of Korean Medical Science, vol. 15, no. 1, pp. 78-82, 2000.

[35] K. Bakker, J. Apelqvist, B. A. Lipsky, J. J. Van Netten, and International Working Group on the Diabetic Foot, "The 2015 IWGDF guidance documents on prevention and management of foot problems in diabetes: development of an evidence-based global consensus," Diabetes/Metabolism Research and Reviews, vol. 32, no. 1, pp. 2-6, 2016.

[36] J. Dros, A. Wewerinke, P. J. Bindels, and H. C. Van Weert, "Accuracy of monofilament testing to diagnose peripheral neuropathy: a systematic review," Annals of Family Medicine, vol. 7, no. 6, pp. 555-558, 2009.
[37] M. Rahman, S. J. Griffin, W. Rathmann, and N. J. Wareham, "How should peripheral neuropathy be assessed in people with diabetes in primary care? A population-based comparison of four measures," Diabetic Medicine, vol. 20, no. 5, pp. 368374,2003

[38] J. Jurado, J. Ybarra, and J. M. Pou, "Isolated use of vibration perception thresholds and Semmes-Weinstein monofilament in diagnosing diabetic polyneuropathy: "the North Catalonia diabetes study"," Nursing Clinics of North America, vol. 42, no. 1, pp. 59-66, 2007.

[39] N. Papanas, A. Gries, E. Maltezos, and R. Zick, "The steel ball-bearing test: a new test for evaluating protective sensation in the diabetic foot," Diabetologia, vol. 49, no. 4, pp. 739-743, 2006.

[40] S. Baraz Pordanjani, N. Sayadi, H. Shahbazian, M. Latifi, and M. Karimi, "Examination with two types of Semmes Weinstein monofilaments as screening tools for diabetic peripheral neuropathy," Iran Journal of Clinical Endocrinology and Metabolism, vol. 14, no. 4, pp. 311-318, 2012.

[41] P. D. Mitchell, "The threshold for protective sensation that prevents neuropathic ulceration on the plantar aspect of the foot: a study of leprosy patients in a rural community in India," Leprosy Review, vol. 72, no. 2, pp. 143-150, 2001.

[42] D. Young, S. Schuerman, K. Flynn, K. Hartig, D. Moss, and B. Altenburger, "Reliability and responsiveness of an 18 site, 10-g monofilament examination for assessment of protective foot sensation," Journal of Geriatric Physical Therapy, vol. 34, no. 2, pp. 95-98, 2011.

[43] J. Booth and M. J. Young, "Differences in the performance of commercially available 10-g monofilaments," Diabetes Care, vol. 23, no. 7, pp. 984-988, 2000.

[44] M. U. Werner, P. Rotboll-Nielsen, and C. EllehuusHilmersson, "Humidity affects the performance of von Frey monofilaments," Acta Anaesthesiologica Scandinavica, vol. 55, no. 5, pp. 577-582, 2011. 


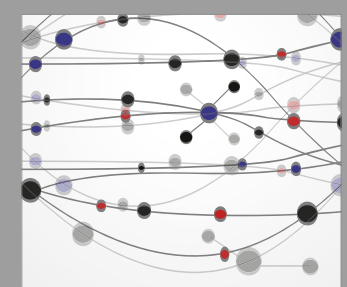

The Scientific World Journal
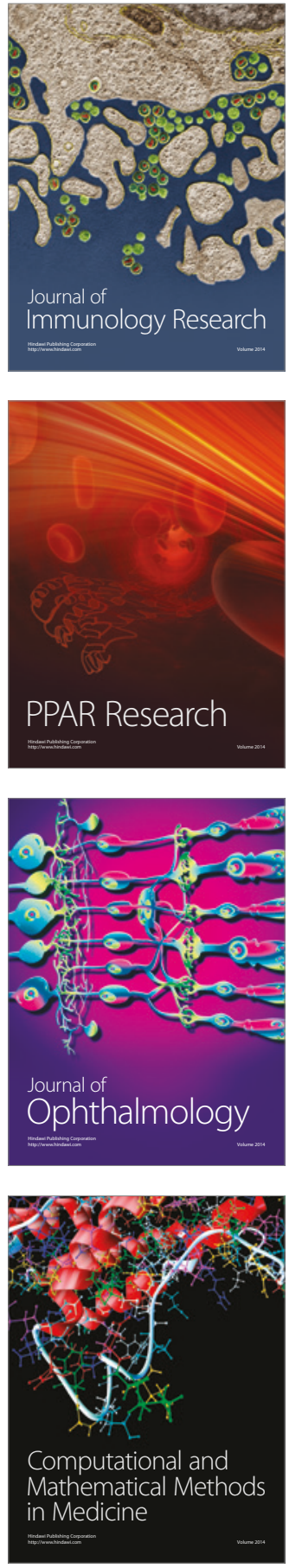

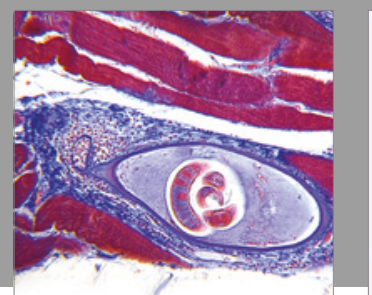

Gastroenterology Research and Practice
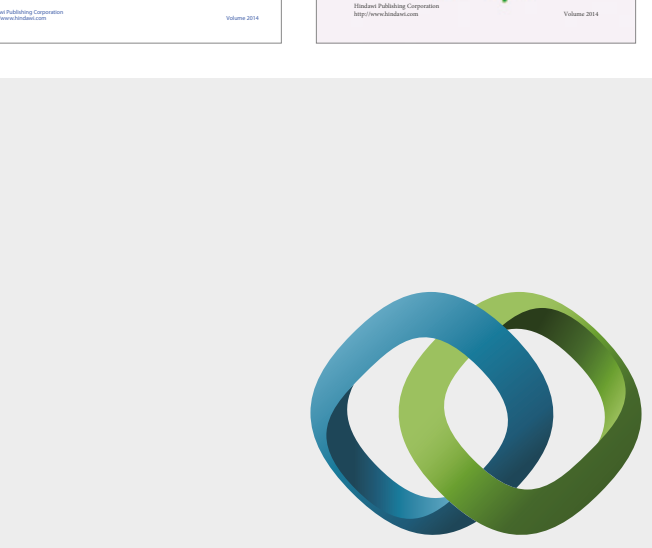

\section{Hindawi}

Submit your manuscripts at

https://www.hindawi.com
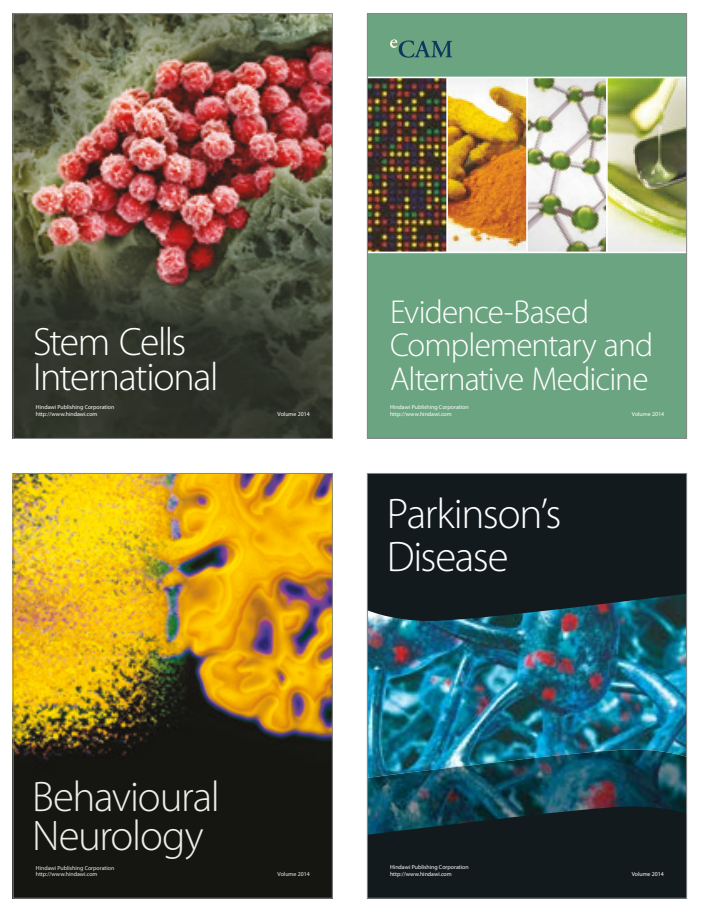
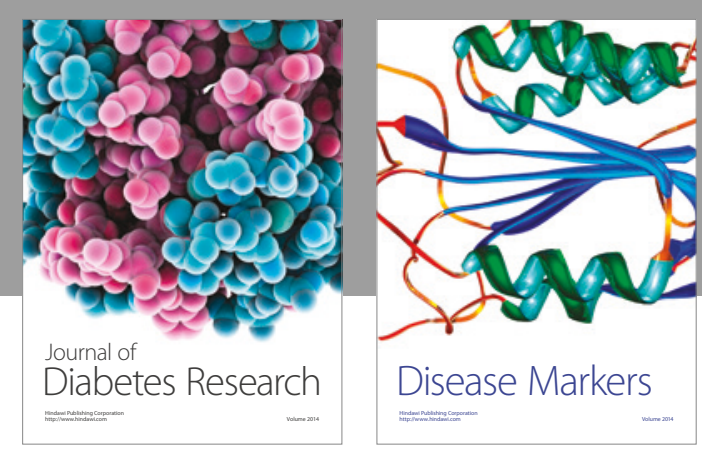

Disease Markers
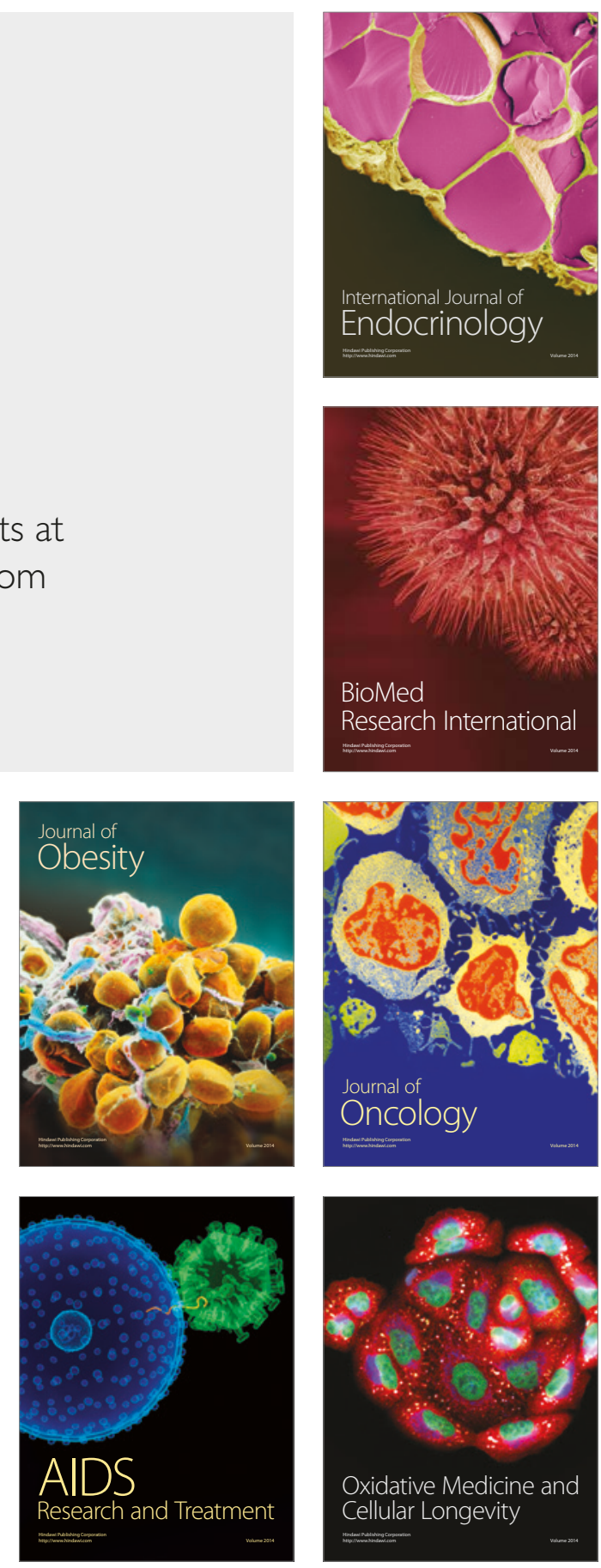